\section{Personal Health, Person-centred Health and Personalised Medicine - Concepts, Consumers, Confusion and Challenges in the Informatics World}

\author{
M. Rigby \\ Keele University, School of Public Policy and Professional Practice, Keele, United Kingdom \\ (also Nordic School of Public Health, Gothenburg, Sweden \\ Dublin City University, Dublin, Ireland)
}

\section{Introduction}

The remit of this paper is to consider how Consumer Health Informatics supports and has a role within the domain of Personal Health Informatics. The initial result is the identification of a set of challenges, not least caused by the taking of familiar words and terms and imbuing them with a new specific meaning within a new focus of health care science, but thereby leaving confusion through duality of old and new meaning. In this process, it is essential to ensure that the new and progressive meaning does not detract from the core values of the generic meaning, and that consumers and non-specialist practitioners do not lose understanding of appropriate health care and reliable health information through confusion of meanings and thus of care delivery. Yet at the same time, it is important for the new science and its related professional practice and consumer information (which should be two facets of the same body of knowledge) to be effectively supported by information science and informatics, and the challenges arising be appropriately addressed.

\section{What do the Words Mean and How are they Used?}

'Personal' is a clearly understood word in common use, and the Oxford Eng- lish Dictionary definitions emphasise the attribution to an individual, and the close link with privacy [1]. Thus the public, patients and consumers could well expect all health care and health records to be 'personal' to the individual, and treated with the appropriate sanctity and confidentiality, unless exceptionally they relate to population health activities. Further, the public could therefore expect a degree of tailoring the individual characteristics.

\section{Personal Health and Personal Health Informatics}

Grouping the words 'Personal Health' together creates a composite concept which further deepens this. Health is itself a personal state, as defined both by dictionary and by the World Health Organisation [2]. Thus 'personal health' must relate to matters pertaining to the health of an individual, with due regard to their biological and familial personalisation, privacy, and circumstances. (As explained below, in the healthcare community a specific facet of this is currently a fast-growing area with the advent of 'personalised medicine' as the application output of deeper understanding of genetics and genomics, but this is threatening to take swamp the broader established concept of personalisation.)

The further composite, 'Personal Health Informatics', brings the application of information and communica 
tions technologies (ICTs) to the support of this core concept of personal state. 'Personal Health Informatics' is the applied science of collection, processing, storing and retrieving data related to the health and health care of an individual, but with the further weight on the 'personal' component to emphasise the personalisation, either by being tailored to the individual or being selected and operated by the individual. This dual-compartment definition thus accommodates ubiquitous and pervasive applications to monitor the vital physiological signs and daily living, and on the other hand commercial devices and Internet applications which the individual uses to find out about their health, healthcare, and related treatment and lifestyle choices.

A search of PubMed, considered the principal scientific source of verified peer-reviewed health science publications, in February 2012 showed no publications with the term "personal health informatics" in the title, though there were four (very disparate) publications in which the three words individually appeared in the title of an entry. Further, there were no entries in which the term "personal health informatics" appeared in the abstract, and only 96 in which all three individual words appeared in any order or construct in the abstract. Thus in considering 'personal health informatics' we are considering a concept which has never been written about scientifically in this specific way. The slightly more eclectic Google Scholar showed globally three papers with "personal health informatics" in the title, and 100 where the phrase appeared anywhere in the text (abstract search not being available). Thus this Yearbook is staking out new ground by focussing on this new formal concept, and this is defined elsewhere [3].

\section{Person-centred Health and Informatics}

Person-centred (or patient-centric) health care and delivery, and informatics applications tailored to this end, are further developments to the logical theme of long-standing aspirations to treat patients as individuals in every sense, in what is often commented otherwise to be 'production line medicine' where the patient fits the system rather than the other way round. Unfortunately this aspiration has often fallen short for logistic reasons as well as provider and professional convenience.

For many years there has been a concept of patient-focussed care, the scientific literature effectively starting in the early 1990s, and indeed it is primarily a phenomenon of that decade, often hospital based and concerned with process re-engineering with efficiency gains as much as on improving the attitude to patients. Patient-centred care seems to be part a deepening, and part a focus on primary and community care, or integrated care linking hospital and home care.

In either form patient focussed or centred care is a relatively modest scientific interest - a search on PubMed for "patient-focussed care" in any context, not specific to informatics, yielded 166 results with the term in the title, increasing to 291 with it in the title or abstract, while "patient-centred care" yielded 100 with the term in the title and 381 if abstracts were searched too; "patient-centric care" added a further 8 and 18 respectively. An overview of this literature shows that 'person-focussed' care had a frequent emphasis on changing the organisation of processes of care, while 'person-centred care' was more likely to have an information or informatics element, partly because of the primary care, community care, or cross-provider integrated care element of the published studies requiring a strong information sharing component, and partly because it is a more recent literature. However, in itself this has no direct link with consumer health informatics.

In the last few years the concept of harmonising the delivery of health care and other forms of personal support which affect and determine health, such as social care and housing support, in order to address health holistically, has arisen. These are recognised as raising new ideas and issues, and are developments on the future agenda for health care development supported by health informatics in an integrated way [4].

\section{Personalised Medicine}

'Personalised medicine' is another term of recent origins, which too carries a specific meaning greater than the sum of its parts. By using the term 'personal' it has strong resonance with 'personal health informatics' and might be expected to share territory; and as it also expects patient understanding and action it has an overlap of intention and methods with consumer health informatics. 'Personalised medicine' tends to be reasonably well understood within the technical arenas of medicine and diagnostics, as being based on genetics and genomics (which confusingly do not appear in its title), but is the cause of considerable misrecognition and challenges when used more widely.

However, when using automated knowledge management systems such as bibliographic searches the trans-Atlantic variants of the English language are a compounding confusion, given the alternate spellings 'personalised' and 'personalized'. A PubMed search yields 58 papers with "personalised medicine" in the title and a further 709 with "personalized medicine", while on searching both title and abstract the figures are 137 and 1904 respectively, giving a combined total for "personalised/personalized medicine" of 767 titles and 2041 titles and abstracts. Of course, the term 'personalised medicine' is not unique in use for genetically-linked care (a confusion caused by its arguably inappropriate adoption as the term for this specific care modality, possibly as a means of seeking to enhance its attractiveness and importance), but certainly from 1999 the genetic-based focus was the focus of publications - giving a more than twenty-fold greater volume of PubMed publications than for 'consumer health informatics' as discussed next. 
The reasons for raising personalised medicine here are three-fold - first, as it becomes more publicised it will hit the public interest and thus lead to a new and different activity within consumer health informatics, bringing its own challenges. Second, it puts further (and at times confused) emphasis on the 'personal' element. And thirdly, it raises significant issues of ethics, and conflicts between privacy and rights based on linkages between autonomous individuals, which may spill over into 'personal health' activities and be articulated within 'consumer health informatics'.

\section{Consumer Health Informatics}

'Consumer Health' and 'Consumer Health Informatics' are also comparatively new terms, and also well established in a short time. Deconstruction of the term down to the individual words is not helpful, given the wide and all-encompassing meanings of 'consumer', while 'health' has already been referred to. However, PubMed showed 36 results on searches for the string "consumer health informatics" being in the title, and 70 when searching title or abstract. The wider tolerance Google Scholar returned 111 publications with "consumer health informatics" in the title, and 1900 with it anywhere in the article. Though relatively modest in size and mainly very recent, this literature includes significant analytic and definitional items from more than a decade ago, including consideration of the issues of consumer health informatics by the United States General Accounting Office in 1996 [5] and the US Agency for Health Policy and Research in 1997 [6], and an early definitive review in the British Medical Journal by Eysenbach in 2000 [7]. Moreover, by contrast with the analyses above on person-centred care and on personalised medicine, this publications count is specifically on informatics papers.

\section{What is Consumer Health Informatics Considered to Be?}

IMIA has a definition of consumer health informatics available from the objectives of its own Consumer Health Informatics Working Group [8]. This states that the Working Group is "concerned with electronic information related to health care available to the public (e.g. Internet, wireless, standalone electronic media)." For its purposes, it adopts the definition of Consumer Health Informatics put forward by the US General Accounting Office in 1996 [5] as "the use of modern computers and telecommunications to support consumers in obtaining information, analyzing unique health care needs and helping them make decisions about their own health". Important in this is the concept that "the consumer interacts with the applications directly with or without the presence of health care professionals", since this is far more than visiting Internet sites for information, and in the modern world of mobile health (mHealth) and of consumer (patient) entries into health records the boundary between consumer health informatics and mainstream health professional informatics becomes blurred.

Eysenbach's early review of the subject [7] took the definition as "Consumer health informatics is the branch of medical informatics that analyses consumers' needs for information; studies and implements methods of making information accessible to consumers; and models and integrates consumers' preferences into medical information systems. Consumer informatics stands at the crossroads of other disciplines, such as nursing informatics, public health, health promotion, health education, library science, and communication science, and is perhaps the most challenging and rapidly expanding field in medical informatics; it is paving the way for health care in the information age." So Eysenbach too, in emphasising the interaction between the consumer and health information and health systems in all forms (and in 2000 less options were available), makes this a challenging and dynamic area, far greater than informational web sites alone.

So in the new era of Personal Health and Personalised Medicine, the all-encompassing Consumer Health Informatics becomes central and highly significant, because it covers any health informatics system by which consumers interact with health systems or obtain information which may influence their health decisions. Thus not just the topic coverage, but issues of accuracy, understanding, ethics, and implications beyond treatments or health lifestyle of the individual are now raised by the fact that the compass of consumer health informatics is changing, and in a significant way. For instance, Alpey et al raise the important issue that consumer understanding is not the same as consumer access, and they consider in detail, in the context of understanding information supplied through web sites, the cognitive and other issues raised [9]. And McDaniel and colleagues from Indiana University School of Nursing were among the first, in 2008, to recognise that the rise in what they refer to as genomic health, more familiarly now called personalised medicine, will radically change consumer health informatics as it interfaces new and complex information sources, and faces new ethical challenges [10].

Thus though the generic definition of consumer health informatics, as shown from its early days, is simple to understand, the implications have also increased radically as health care and technology have got more complex at a time when consumer empowerment and information access have grown significantly. It is now difficult to think of consumer health informatics as a single entity, as the different technical and content manifestations lead to many very different sources and types of information, and in turn raise their own issues of understanding, safety, and ethics. 


\section{Manifestations of Consumer Health Informatics}

The nature, content, and available communications vehicles of consumer health informatics have changed significantly in the last two decades. Initially the focus was very largely on Advice, Choice and Information. The purpose was to enable citizens to know more about health and its determinants, in order to be able to make better lifestyle choices, and to be able to understand specific conditions and their management. This was very Internet focussed, as citizens became familiar with the rapidly expanding world wide web, and was part of the drive for citizens to take more responsibility for managing their own health.

In turn, this progressed in two directions, one being to focus on treatments as much as on diseases, and the search for the best treatment - or the newest treatment claims. In parallel, consumer health informatics moved to providing information about care providers of many kinds including hospitals, and raised issues of how to identify quality not least for complex conditions or linked to comorbidity.

With the wider advent of web logs (blogs) and chat rooms came the development of self-help groups - on line communities which share experiences, advice, and ideas. These have provided valuable guidance and support to many people. This has now further developed into dedicated active initiatives such as Patientslikeme [11] - a now widespread consumer health initiative which is open and explicit on three paradigm-changing aspects - in being led by citizens who are technologists not health practitioners; in seeking to improve health systems themselves; and is for profit. This is a significant move on from health organisations making consumer information available on their institutional sites, and voluntary patient groups having a web site as their 'shop window'.

\section{Retail 'Productisation'}

A second more recent development is that of production of products for consumers to monitor their health. Here the threshold of consumer health informatics is even more difficult to define. Some tools, such as blood pressure monitors, blood glucose monitors, and devices to identify women's fertile periods are well established and might be considered 'pre-informatics' as there is no communication or computing element involved other than electronic calculation of a simple reading based on an internal algorithm.

The new situation will arise as these retail devices become more complex in their calculations, depend on reference data sets which are not identified, or contain an element of personalisation in their calculations based on individual medical history. If they are stand-alone then they are likely to be considered retail medical devices, but if they interlink through electronic communications with personal records or vital sign monitoring subsets, health care providers, reference databases, or advice-providing sites they must be considered a part of consumer health informatics in that they will be informatics devices used by consumers of their own autonomous instigation.

\section{Record Access}

A further new dimension affecting consumer health informatics is access to personal health records. This concept enables patients to access their own electronic health records, usually through their primary care provider. Initially a result of campaigns to empower patients, this concept has recently gained momentum. It is now policy of the European Commission, with Action 75 of the Digital Agenda for Europe being to give European citizens secure online access to their medical health data and achieve widespread telemedicine deployment [12]. It is now a policy intention in the UK, but has already been achieved in Denmark
[13]. This brings consumer health informatics into interface with the mainstream of health care and its delivery, in that the consumer function is a portal to the main provider record - no longer is consumer use of health informatics a separate arena, but in this functionality is an aspect of mainstream informatics.

\section{Information Seeking; Information Providing}

This transition in consumer health informatics activities, from being solely information receiving, to in many cases being information providing, moves the citizen user of health informatics facilities from external non-involved viewer who simply acts on the information they view, to that of co-creator - whether this is through contribution to patient experience sites or to commenting on their own provider record. However, within consumer health informatics this co-creating is of system or lifestyle knowledge, outside the treatment dialogue. The consumer may now be not just simply reading and acting, but feeding back, though this is explicitly not within the patient-clinician relationship and the health record.

For the purpose of this review a clear divide has been identified - to exclude from consumer health informatics any activities which are related to a specific episode of treatment, whether the consumer or patient involvement is by passive or active monitoring, telemedicine, or structured planned data entry. Such activities are part of a clinician-led or clinician-partnered activity, and thus fall outside consumer health informatics. Specifically, consumer health informatics is taken as being consumer initiated and not as a specific therapeutic course of treatment or support (specific clinical conditions may be involved, but consumer health activities are outside the clinical treatment programme).

There may be apparent borderline areas, such as consumer feedback on a care provider, but not involving the 
treatment dynamic. In summary, the participant in consumer health informatics is acting in a citizen role; when participating in a treatment or monitoring transaction led by a professional care provider they are acting in a patient role.

\section{The Consumer and Personalisation of Care}

The issue that has not been well identified by the various pressures that have promoted 'personalised health' as genetically based is that there has been a long drive to seek to personalise health care and its delivery, in line with core human values, but this has not been seen as a major priority until now, by which time all the terms and labels have been appropriated. In general terms this personalisation has applied from the time of Hippocrates and even earlier within health professional practice. Within computerisation and health informatics, the personal privacy through 'isolation' of individual records by means of data protection and confidentiality has been a core principle, but much less so the 'personalisation' through recording of personal aspects and values (as being distinct from biometric values). One exception is that the Medical Informatics Europe (MIE) conference in 1994 took as its theme 'Human Values in Medical Informatics', which enabled integrational papers on human values of patients and staff in electronic records, and resultant person-sensitive care, such as [14].

Fierz writing in Switzerland in 2004 summed this up well - scientific medicine has in its enthusiasm for objectivity partly neglected the long-established aim of personalising all medicine, even though it is known that 'one fits all' does not work well in medicine; however, the time for change is ripe in the post genomic era, and with modern information and communications technology in support - "the goal is magnificent: personal health planning, early diagnosis, the right drug for the right patient, and predictable side effects" [15].

Though therefore the very nature of health care and its record keeping is personalised - the personal history, and the personal and very confidential record are cornerstones - too often the care delivery itself is formulaic in its construct, and only personalised in the sense of fitting a treatment protocol to the diagnostically-defined problem, rather than the problem as manifest in the person's life and lifestyle. Indeed, this mechanical element, and the standardised delivery within an efficiency framework, has been a trigger for elements of consumer health mobilisation, as patients seek to express themselves and their aspirations as people [11].

\section{Personalised Care Planning}

Care planning is a well-established means within nursing and many other health professions other than medicine for ensuring that the delivery of care meets the individual personal needs and circumstances of patients, and has a strong literature in this context, such as [16]. The result is that care delivered is as personalised as possible, and health informatics should ensure this, such as reported in [14].

The same personalisation can be seen as an objective of electronic health records (EHR), especially in specific domains such as mental health, making the EHR the vehicle for ensuring personal and personalised care [17]. Indeed, a literature analysis from this same care domain of mental health shows that the illness itself is the subject of personalisation to the individual [18], yet this is only a deepening of the recognition in care planning that the effect of morbidity on the individual is personal to their pattern of daily living and activities, and treatments and their delivery should similarly be personalised. The same applies to other health and morbidity domains.

\section{Personalised Medicines}

It is not just overall care which should be personalised in its planning, but also medicines specifically. The current pattern of administering standard doses is crude. The benefits of personalising medicines themselves are demonstrable [19]. A further form of personalisation is bio-identical hormone therapy [20]; here the composition of the pharmaceutical product rather than the dose or administration is personalised.

\section{Personalising E-Health}

With not only an increasing number of ways in which health care can and should be personalised, and increasing consumer knowledge and expectations, it is natural and appropriate that ehealth should be used to achieve a data and process dependent activity of some complexity. Apart from the use of electronic records as exemplified in [14] and [17], from Greece comes recognition of the social utility of personalised e-health in delivering personalised care [21].

These are examples of recognition of the inter-linkage between personalised health records and personalised care; the health record architecture is generic and the data are the means of personalisation. However, in the preceding issue of this Yearbook Kalra has raised the next stage of personalisation, that of the personalised functions of Web 3 and thus Health Informatics 3 [22].

\section{So Where Does that Leave Consumer Health Informatics?}

Consumer health informatics became a significant field with the advent of large-scale public access to the Internet. This itself was an important step in empowering citizens with health information, enabling them to better manage their own health (both in healthy lifestyles and in response to disease), 
and to start to even up the power imbalance between health professional and patient.

Apart from the Internet, two new technical developments have added to the scene, enriching it yet raising their own new issues and blurred boundaries. One of these, access (including from home) to individual health records, and related to that remote monitoring for a specific condition, provided by a healthcare or similar agency, is already explained as being excluded from the definition of consumer health informatics adopted here, as it is a more patient-friendly interface with the care process, rather than seeking of generic information - though to paraphrase the Amazon approach to on-line consumers, 'if you liked record access, you may like information access'.

\section{Consumer Initiated e-Health}

The process of crossing the line from interacting with a personal EHR, to initiating self-management using ehealth, leads to a new field for consumer health informatics. An Australian group has identified the potential value for consumers of engaging in ehealth for their own self-management, but also that this area is significantly under-researched [23], while simultaneously a Dutch group sees this approach as an empowerment means, but also identifies the lack of research [24]. So clearly this is a potentially important area for future development needing further scientific study.

\section{Social Media}

A second new phenomenon that is a potential major methodology for consumer health informatics is social media. Blogs and chat rooms give a whole new dynamic to supportive groups such as disease-specific groups, and enable feedback and discussion with formal healthcare providers. And as indicated earlier, new sites have built up based on the new social media [11]. Sites such as Facebook, meanwhile, though not health-specific, are a valuable communications conduit for health interests as well as sites on which individuals share details and seek support.

\section{YouTube}

One particularly interesting, and on reflection challenging, social medium being used in health is YouTube. Though usually thought of as a means of exchanging lighter short videos, such as amusing or candid camera clips, music or family scenes, it is now being used formally within health, not least because it provides a good way of streaming video into any house where a citizen may need to acquire skills or dynamic knowledge, and is now being considered as a health professional aid when advising citizens $[25,26]$.

However, there is a big difference, probably not appreciated by the average consumer, between following a posted link and general searching. For instance, at the time of preparing this paper, searching on the YouTube site on "infantile spasms" yielded 704 video clips ranging from explanatory ones to those from parents effectively asking for a diagnosis, or at least whether their child was 'normal'. Searching "suicide" yielded 291000 results, ranging from offers to help regain a healthy state of mind to clips (among the first results) of the act allegedly taking place - clearly largely inappropriate postings though with some of potential value. By contrast "condom use" yielded 8450 results, of which the great majority of the first hits were responsible advice which it could be difficult for young people to gain by any other means.

One of the challenges when viewing or using You Tube is the linked offers which are made adjacent to the viewing screen, the advertisements which scroll across the screen or precede running of clips, and the sug- gested further viewing. Unless controls are placed to protect direct hypertextlinked clips from this intrusion, the use of this medium becomes dangerous particularly for health topics. This is less of a challenge when a hypertext link is supplied by a recognised agency, but is a serious challenge and restriction on effectiveness in a healthcare or behavioural setting when using the free search facility - in other words, by serious-minded thinking consumers.

\section{Consumer Health Informatics in the Personal Health and Personalised Medicine Era}

So as health informatics and personalised medicine both expand and become more citizen-friendly and accessible, what are the issues for consumer health informatics? The main effects are likely to be increased demand and expectation, coinciding with the availability of new media. And conversely, expectations and ideas gained though consumer health informatics may increase pressure for personalised medicine services, as well as for personalisation of care delivery.

It will be important to see what phenomena and sites emerge in the personalised medicine area of the consumer health informatics media. Certainly there is a risk of exploitation of anxious people, and of sale of services of doubtful validity, as some citizens seek to allay anxieties which may or may not be well-founded about their personal risk. It cannot be assumed that all those active in the healthcare market are ethical - if it is now necessary to remove rhinoceros horns from museums across Europe as they are sought by thieves to meet a demand for spurious medicinal value [27], what other false claim based products will be placed in front of gullible worried individuals?

A further issue which is likely to increase with the popularisation of per- 
sonalised medicine is that of the calling into question of assumed parentage. The obtaining, quite legitimately, of a genetic profile, linked to accessing Internet information on conditions, inheritance, and relative risks, can easily be seen to have the potential to lead to very damaging situations, if genomic profiles of close family members and consequent recommended clinical action are seen to be incompatible with believed consanguinity relationships. There are likely to be cases of stable family situations where parenthood is not as has been supposed - either because of an early remarriage which has not been revealed to a child, an undisclosed adoption, egg or sperm donorship from which the commissioning couple has always considered the child in every sense as 'theirs', a genuine lack of knowledge of parentage (as made light of in 'Mama Mia'), or an infidelity by a wife many years ago and hitherto not suspected by any other party. There is no easy answer, as loving and trusting relationships may be severely challenged, yet at the same time any individual has the right to ascertain their own genetic history, and the Internet does not have the same sensitivity of approach in revealing facts or knowledge as a trained individual.

As for the more generic personalisation of health, consumer health informatics should be an enabling force. Whether by providing advice about what types and choices of care should be offered, or giving information about providers, web-based information should be helpful and empowering. The key issue will be ensuring that each citizen involved finds the right, accurate and intelligible, information.

\section{Challenges and Issues}

So as consumer health informatics moves forward into a new era of personal health and personalised medicine, some old challenges remain and new ones arise.

\section{Authenticity and Accuracy}

The challenge of ensuring the authenticity and accuracy of Internet sites has long been a concern. Authenticity relates to whether the organisation is what it seems to be - for instance, when the names of formal organisations are parodied with only a small variant, leading a user to believe they are reading an official site when they are not. A number of organisations have problems with unofficial 'shadows'; the citizen may or may not spot the difference.

Accuracy refers to the content. There has for a long time been a concern about the lack of verification of site content, leaving the consumer to differentiate robust from less authentic or validated material. The Health on the Net Foundation has established a long-recognised Code, but this is voluntary, and for a considerable period was a set of principles based on self-regulation though with no enforcement mechanism. In 1997 a workshop was organised in Turku, Finland, to consider the risks of lack of certification of e-health services or internet sites, and largely as a result in 1999 the European Commission funded the project Towards European Accreditation and Certification of Health Informatics services (TEAC Health). This recommended among other actions a voluntary system of third party validated verification seals for Internet sites [28, 29, 30], and subsequently the Commission adopted a policy of sponsoring such a system, but failures of the funding mechanism for projects resulted in this policy not being fulfilled. This concept has now been developed by the Health on the Net Foundation, and their HON Code seal is now provided as a certified web seal [31], and this has been studied $[32,33]$.

\section{Equity of Access}

The more that consumer health informatics becomes acknowledged, particularly by health care providers, the greater will be the expectation that citizens will be expected to use it as part of managing their health. This is commendable, but does raise the serious risk of the creation of a 'digital divide', whereby the more affluent and educated sectors of the population have good access, while the poorer and marginalised section, and the older elderly, have less access - and these are the sectors of the population most likely to have health problems.

\section{Effectiveness and Safety of Use}

Similar to equity of access, the issues of effectiveness and safety of use are likely to create a population gradient, and one skewed in the opposite way to need. Many older or less formally educated members of society will not be digitally fluent. And a proportion of those who use the internet in straightforward ways, such as for on line purchases, chatting, or social media, will not be skilled when it comes to structured searching, and filtering of the reliable from the less reliable, especially as the most significant scientific material may be in less accessible language; furthermore, such users may not realise their own vulnerability due to their successful use of the Internet for simpler activities. There are a number of attempts to increase digital literacy and health literacy, but they are currently small when matched against the size of the need for increasing health-specific digital literacy across all sectors of society globally.

\section{Enablement}

Structured enablement is one way to encourage wider and more fluent use of consumer health informatics. This would involve trusted centres or individuals offering facilities and advice to individuals wishing to use the Internet for health purposes. It could be through health facilities, such as primary care centres, or through public libraries where supported Internet access is provided. However, so far this is a need which has not been identified or ad- 
dressed, valuable though it could be in a more consumer-orientated and information-based society.

\section{Impersonation, Grooming and Fraud}

Probably because health workers are positively motivated, little thought seems to have been given to anticipating and tackling malevolent exploitation of consumer health informatics and its users. In wider Internet usage attempts to obtain banking details though bogus e-mails, or obtain personal details such as by claiming an e-mail account is full or in conjunction with a spurious investment or lottery win, are commonplace. On line retail sales of products with mythical powers of cure are known about, as are sales of low efficacy substitutes for effective medications. And in normal society, attempts to skim bank cards, or to gain entry to older people's houses by posing as utility engineers or public officials, and not unusual. Chat rooms are vulnerable to individuals who will project false persona so as to befriend a type of person of their choice. So logically, consumer health informatics is a ripe field for that tiny but determined minority of society who will go to great lengths to defraud or exploit - within its user community there will be less digitally experienced persons who are very worried about a health condition, who may be vulnerable to being easily talked into pointless expensive purchases, to joining exploitative self-help groups, or even to meeting one-to-one to share experiences or get help. So far there is little evidence of this happening, but as consumer health informatics expands, and more people seek on line support, it is surely inevitable that the rogue community anticipate this and start to become active. The health informatics community must anticipate this, and start to advise both standards, and consumer education and best practice warnings. Anticipation and prevention are important approaches to health; they should apply in this domain as much as any other.

\section{Retail Medical Devices}

Finally, as product types and markets expand, so will retail products. The cross-over from being a consumer product to being a consumer health informatics retail device, such as when the product communicates with an analytic centre or reference database, is not well defined. This debate links also to the issue of when software or informatics products become medical devices, and in both the United States and Europe this is currently a discussion and development area [34]. This too needs to be an area of proactive debate and initiatives.

\section{The Need for Action}

Consumer health informatics has for over a decade had a clear definition and role. However, the welcome extension of consumer orientated approaches to health and healthcare, aided and stimulated by other new concepts such as personalisation of care, 'smartening' of care [35], social media and other participatory phenomena, new retail products, and personalisation of medicine, will lead to a new era. This will be more complex because of the greater personal interaction with sites, and that this is by definition a personal and private area of activity. Involvement will spread from the more able and educated sections of society to a wider cross-section including more vulnerable people. At the same time, this widening will raise challenges both of widening of issues and risks such as simple misinterpretation, to the opening up of interest by the malevolently exploitational. And as e-health is global this ceases to be a local or national issue but becomes global, as with hacking and phishing.

The consumer health informatics community should also initiate a more active programme of enablement as part of facilitating deeper and better use, and acting against the digital di- vide as well as traditional health inequalities. This would include facilitating consumer understanding and awareness through educational and promotional programmes, and stimulating and promoting models of supported access and use.

Overall, the global health informatics community needs to give the area of consumer health informatics deeper attention. The potential benefits, and the potential risks, are going to multiply, and no longer should institutional health provider informatics be seen as the sole area of major importance. At the same time this is not an area where health informatics should feel forced to work alone. Other competent organisations already active in aspects of or related to health informatics include the World Health Organisation, the International Telecommunications Union, the World Trade Organisation, and crime prevention and detection networks. IMIA should establish a role in seeking to bring these organisations together to address proactively the issues of promoting consumer health informatics in a positive yet protected way. Included in innovations which should be developed jointly, with a high profile the world citizen should recognise and trust, are third party verified seals for sites and services (as already applies in site security and financial transactions), and simple education and advice programmes on how to find and use consumer health sites.

\section{Conclusion}

As information and communication technologies continue to expand in functionality, coverage and uptake, so this coincides with and facilitates the move to greater consumer orientation and personalisation of health care, and emphasis on personal responsibility for health. Consumer health informatics can and should play an important role in this; and it should enable the citizen 
to benefit from such facilities in an informed, effective and safe way. Recognition of the growing importance of the sector must also lead to reluctant acceptance that the greater its value and use, the greater the attraction for malevolent exploitation of its users, with a concomitant need to take socially responsible preventive action.

Unlike many other areas of health informatics, consumer health informatics is not an organised sector, and has no corporate organisation or affinity. IMIA is the lead informatics body, but does not act solely for health, communications, nor citizen safety. The personalisation of other aspects of care should be a trigger for recognition of the need for concerted action to protect safe personalisation of health through consumer health informatics.

\section{References}

1. Compact Oxford English Dictionary, Oxford: Oxford University Press, 3rd. edition; 2005.

2. World Health Organisation. Constitution of the World Health Organization, in Basic Documents, Forty-fifth edition, Supplement, October 2006, WHO; Geneva.

3. Geissbuhler A, Kulikowski C. Personal Health Informatics. Editorial. Yearb Med Inform 2012:2-3.

4. Rigby M, Hill P, Koch S, Keeling D. Social Care Informatics as an Essential Part of Holistic Health Care: A Call for Action; Int J Med Inform 2011;80:544-54.

5. United States General Accounting Office. Consumer health informatics: emerging issues. Washington, DC: US Government Printing Office; 1996. (No T-AIMD-96-134.)

6. Hersey JC, Matheson J, Lohr KN. Consumer health informatics and patient decision-making. Rockville, MD: Agency for Health Care Policy and Research;1997. (AHCPR research report No 98-N001.)

7. Eysenbach G. Consumer Health Informatics. BMJ 2000;320:1713.

8. http://www.imia-medinfo.org/new2/node/137 (accessed 18 January 2012)

9. Alpay L, Verhoef J, Xie B, Te'eni D, Zwetsloot-
Schonk JH. Current Challenge in Consumer Health Informatics: Bridging the Gap between Access to Information and Information Understanding; Biomed Inform Insights 2009 Jan 1;2(1):1-10.

10. McDaniel AM, Schutte DL, Keller LO. Nursing Outlook 2008 Sep-Oct; 56(5):216-223.e3. Review.

11. http://www.patientslikeme.com/(Accessed 20 April 2012)

12. http://ec.europa.eu/information_society/newsroom/ cf/fiche-dae.cfm?action_id=233\&pillar_id= 49\&action=Action $\% 2075 \% 3 \mathrm{~A} \% 20$ Give $\% 20$ Europeans $\%$ 20 secure $\% 20$ online $\% 20$ access $\% 20$ to $\% 20$ their $\%$ 20 medical $\% 20$ health $\% 20$ data

13. https://www.sundhed.dk/

14. Rigby MJ, Robins SC. Human Values as the Core Focus of Community Health Computing. In Barahona P, Veloso M, Bryant J: Proceedings of the Twelfth International Congress of the European Federation of Medical Informatics, Lisbon, Portugal, 22-26 May 1994; Portuguese Association for Medical Informatics, Lisbon; 1994.

15. Fierz W. Challenge of personalized health care: to what extent is medicine already individualized and what are the future trends? Med Sci Monit 2004 May;10(5):RA111-23. Epub 2004 Apr 28.

16. Morton T, Morgan M. Examining how personalised care planning can help patients with long-term conditions. Nursing Times 2009 Sep 22-28;105 (37):13-5.

17. Mooney M, Barry S, Draper R. The electronic patient record as a guarantor of personalised mental health care. Stud Health Technol Inform 1998;52 Pt 1:14-7.

18. Bolton D. Conceptualisation of mental disorder and its personal meanings. J Ment Health 2010 Aug;19(4):328-36

19. Crommelin DJ, Storm G, Luijten P. ,Personalised medicine" through, personalised medicines": time to integrate advanced, non-invasive imaging approaches and smart drug delivery systems, Int J Pharm 2011 Aug 30;415(1-2):5-8. Epub 2011 Feb 12.

20. Romero M. Bioidentical hormone replacement therapy. Customizing care for perimenopausal and menopausal women. Advanced Nurse Practitioner 2002 Nov; $10(11): 47-8,51-2$.

21. Kadda A. Social utility of personalised e-health services: the study of home-based healthcare. Int J Electron Healthc 2010;5(4):403-13.

22. Kalra D. Health informatics 3.0; Yearb Med Inform 2011;6 (1):8-14

23. Hordern A, Georgiou A, Whetton S, Prgomet M. Consumer e-health: an overview of research evidence and implications for future policy. HIM Journal 2011;40(2):6-14.

24. Alpay L, van der Boog P, Dumaij A. An empowerment-based approach to developing innovative e-health tools for self-management. Health Informatics J 2011 Dec;17(4):247-55.
25. Murugiah K, Vallakati A, Rajput K, Sood A, Challa NR. YouTube as a source of information on cardiopulmonary resuscitation. Resuscitation 2011 Mar;82(3):332-4. Epub 2010 Dec 24.

26. Lim Fat MJ, Doja A, Barrowman N, Sell E. YouTube Videos as a Teaching Tool and Patient Resource for Infantile Spasms. J Child Neurol 2011 Jul;26(7):804-9. Epub 2011 May 6.

27. http://www.spiegel.de/international/europe/ 0,1518,821132,00.html (accessed 14 March 2012)

28. Forsström J, Rigby M, Roberts R, Nilssen S-I, Wyatt J, Beier B, et al. Towards Evaluation and Certification of Telematics Services for Health (TEAC-Health) - Key Recommendations (Final Report of the EU Health Telematics Application Programme project HC 4101, Towards European Accreditation and Certification in Health (TEACHealth)); University of Turku, Turku, Finland; 1999.

29. Forsström J, Rigby M. TEAC-Health - Researchbased Recommendations for European Certification of Health Telematics Services. In: Hasman A, Blobel B, Dudeck D, Engelbrecht R, Gell G, Prokosch H-U: Medical Infobahn for Europe: Proceedings of MIE2000 and GMDS2000, Amsterdam: IOS Press; 2000.

30. Rigby M, Forsström J, Roberts R, Wyatt J.Verifying Quality and Safety in Health Informatics Services; BMJ 2001;323(7312):552-6.

31. www.hon.ch (accessed 20 April 2012)

32. Laversin S, Baujard V, Gaudinat A, Simonet MA, Boyer C. Improving the transparency of health information found on the internet through the honcode: a comparative study. Stud Health Technol Inform 2011;169:654-8.

33. Boyer C, Baujard V, Geissbuhler A. Evolution of health web certification through the HONcode experience. Stud Health Technol Inform 2011;169:53-7.

34. Talmon J, Rigby M, Pelayo S, Ammenwerth E. The Medical Device Directive and Software: The implications; in Moen A, Andersen SK, Aarts J, Hurlen P, editors. 23rd International Conference of the European Federation for Medical Informatics User Centred Networked Health Care, MIE 2011 / CD / Panels

35. www.oecd.org/sti/smarterhealth (Accessed 20 April 2010)

Correspondence to:

Emeritus Professor Michael Rigby

Lavender Hill

6 Carrighill Lower, Calverstown

Kilcullen, C0. Kildare, Ireland

Tel: +35345485858

E-mail: m.j.rigby@keele.ac.uk 